\title{
A Case Report of Primary Mucinous Adenocarcinoma of Urinary Bladder with Review of Literature
}

\author{
Umesh Das ${ }^{1, *}$, Vishwanath Sathyanarayanan ${ }^{1}$, Lakshmaiah K.C. ${ }^{1}$, Champaka ${ }^{2}$, Lokanatha $D^{1}$, Suresh TM $^{1}$, \\ Linu Jacob ${ }^{1}$, Suresh Babu ${ }^{1}$, Govind Babu K ${ }^{1}$ \\ ${ }^{1}$ Department of Medical Oncology, Kidwai Memorial Institute of Oncology, Bangalore, Karnataka, India \\ ${ }^{2}$ Department of Pathology, Kidwai Memorial Institute of Oncology, Bangalore, Karnataka, India \\ *Corresponding author: drumesh.das07@gmail.com
}

Received October 29, 2013; Revised November 09, 2013; Accepted January 10, 2014

\begin{abstract}
The primary mucinous adenocarcinoma of the bladder is an extremely rare urologic entity, which is less than $2 \%$ of all urinary bladder tumours and often presents in advanced stage. A 68-year old gentleman was diagnosed with a primary mucinous adenocarcinoma of the bladder from biopsy of anterior abdominal wall ulcer. Immunohistochemistry was done to rule out metastasis from colon and prostate and it confirmed the nature of the tumour. As the patient presented in advanced (stage IV), palliative intent chemotherapy with gemcitabine and cisplatin was administered, however he succumbed to the illness after one cycle of chemotherapy. The primary adenocarcinoma of urinary bladder creates a diagnostic dilemma, as it is difficult to differentiate metastatic adenocarcinoma of the colon and prostate. The immunohistochemistry has a leading role, aiding in diagnosis.
\end{abstract}

Keywords: mucinous, adenocarcinoma, urinary bladder

Cite This Article: Umesh Das, Vishwanath Sathyanarayanan, Lakshmaiah K.C., Champaka G, Lokanatha D, Suresh TM, Linu Jacob, Suresh Babu, and Govind Babu K, "A Case Report of Primary Mucinous Adenocarcinoma of Urinary Bladder with Review of Literature." American Journal of Clinical Medicine Research 2, no. 1 (2014): 1-3. doi: 10.12691/ajcmr-2-1-1.

\section{Introduction}

Urinary bladder cancer is the second most frequent tumor of the genitourinary tract. [1] Adenocarcinomas account for less than $2 \%$ of all bladder cancers. [2] The metastatic carcinoma is the most common type of adenocarcinoma of urinary bladder. The primary sites for these tumors include the rectum, stomach, endometrium, breast, prostate, and ovaries. Primary mucinous adenocarcinoma is a rare urological entity. We present this rare case with review of the current literature.

\section{Case Report}

68 year old gentleman, an agriculturist, from rural southern India presented with a ulcerative lesion in the infraumblical region of 2 months duration which was associated with pain and sero sanguineous discharge. He also gave history of haematuria which was intermittent without clots and painless. However there was no history of irritative or obstructive symptoms during micturition. He had lost five kilograms of weight over last 3 months and was also having anorexia. He denied history of pain abdomen, nausea, vomiting, haematemesis or malaena. He also denied history of smoking or ethanol consumption or history of drug abuse. His past history was unremarkable. On examination, he had Eastern cooperative oncology group (ECOG) performance status (PS) of one and was pale. There was no evidence of palpable peripheral lymphadenopathy. His vitals were unremarkable. Two ulcero proliferative lesions with granulation tissue were seen on inspection of the abdomen. (Figure 1). Palpation revealed $8 \times 7 \mathrm{~cm}$ midline mass extending from the umbilicus to the suprapubic region with induration over the ulcerative area. There was no hepatomegaly or splenomegaly. Digital rectal, chest and heart examinations were unremarkable. On investigating, his haemoglobin was $11.6 \mathrm{~g} \%$, white count of 9400/cumm and thrombocyte count of 294000/cumm. His comprehensive metabolic profile revealed random blood sugar, renal functions and liver function tests with in the reference range. His chest roentgenogram, 12 lead electrocardiogram and echocardiogram did not show any abnormality. His serum for human immunodeficiency virus (HIV) and Hepatitis B (HBsAg) were negative. Esophago-gastro-duodenoscopy and colonoscopy were normal. Serum Carcino embryonic antigen (CEA), carbohydrate antigen 19-9 (CA 19-9) and prostate specific antigen (PSA) were within reference levels. A computed tomography (CT) of the abdomen and pelvis showed a 7.4 x $7.7 \mathrm{~cm}$ sized ill defined heterogeneous mass lesion in the anterior aspect of the dome of urinary bladder with irregular calcification in the peripheral area (Figure 2). There was also an endophytic growth projecting into the urinary bladder and an exophytic component causing infiltration of the anterior abdominal wall. Findings under cystoscopy showed a sessile mucinous growth with increased vascularity seen 
in the dome of the bladder with a normal ureteric orifice and base of bladder. Histopathological study of the anterior abdominal wall ulcer biopsy showed mucin secreting adenocarcinoma of the bladder with a negative
PSA on IHC (Figure 3). As the patient presented in advanced (stage IV), palliative intent chemotherapy with gemcitabine and cisplatin was administered, however he succumbed to the illness after one cycle of chemotherapy.

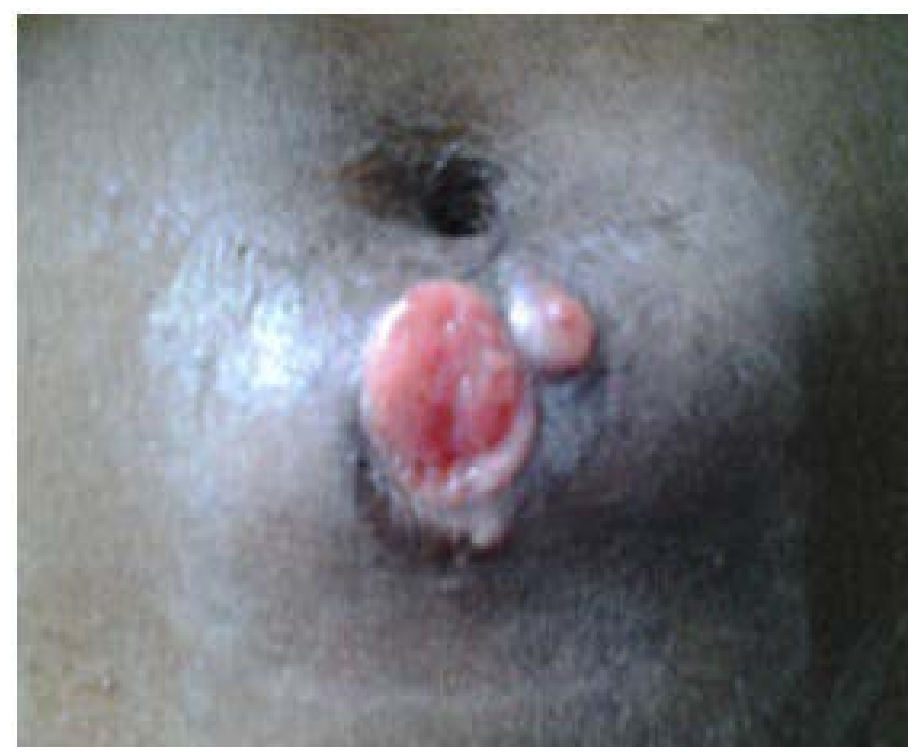

Figure 1. Showing ulcero- proliferative growth $(\rightarrow)$ in the infra - umbilical region
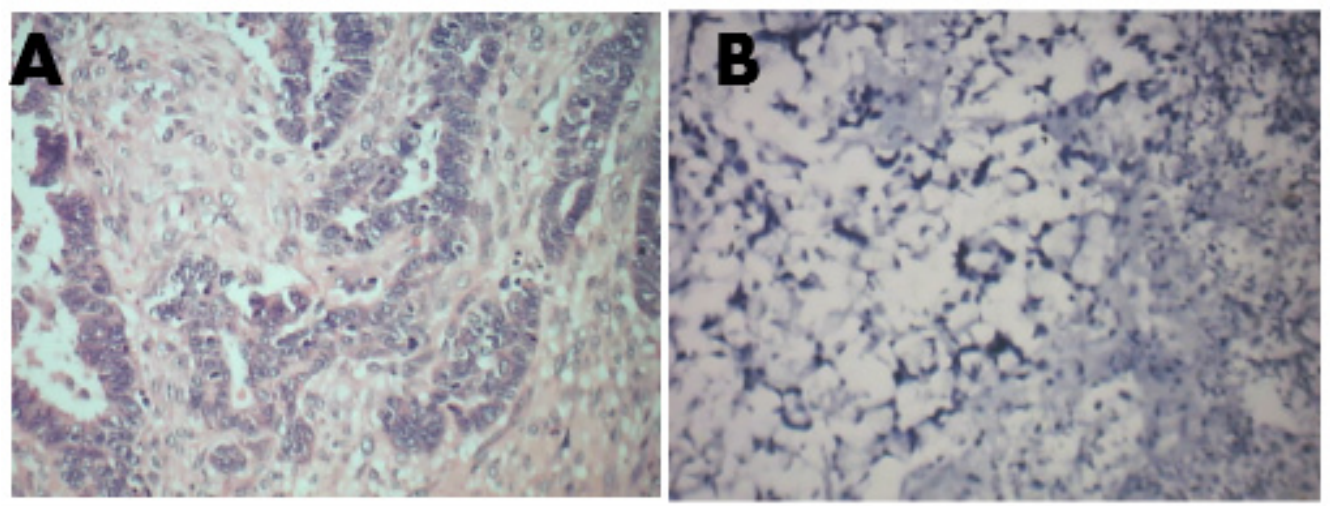

Figure 2. CT Abdomen and Pelvis showing Ill defined heterogeneous mass lesion $(\rightarrow)$ in the anterior aspect of the dome of urinary bladder with irregular calcification

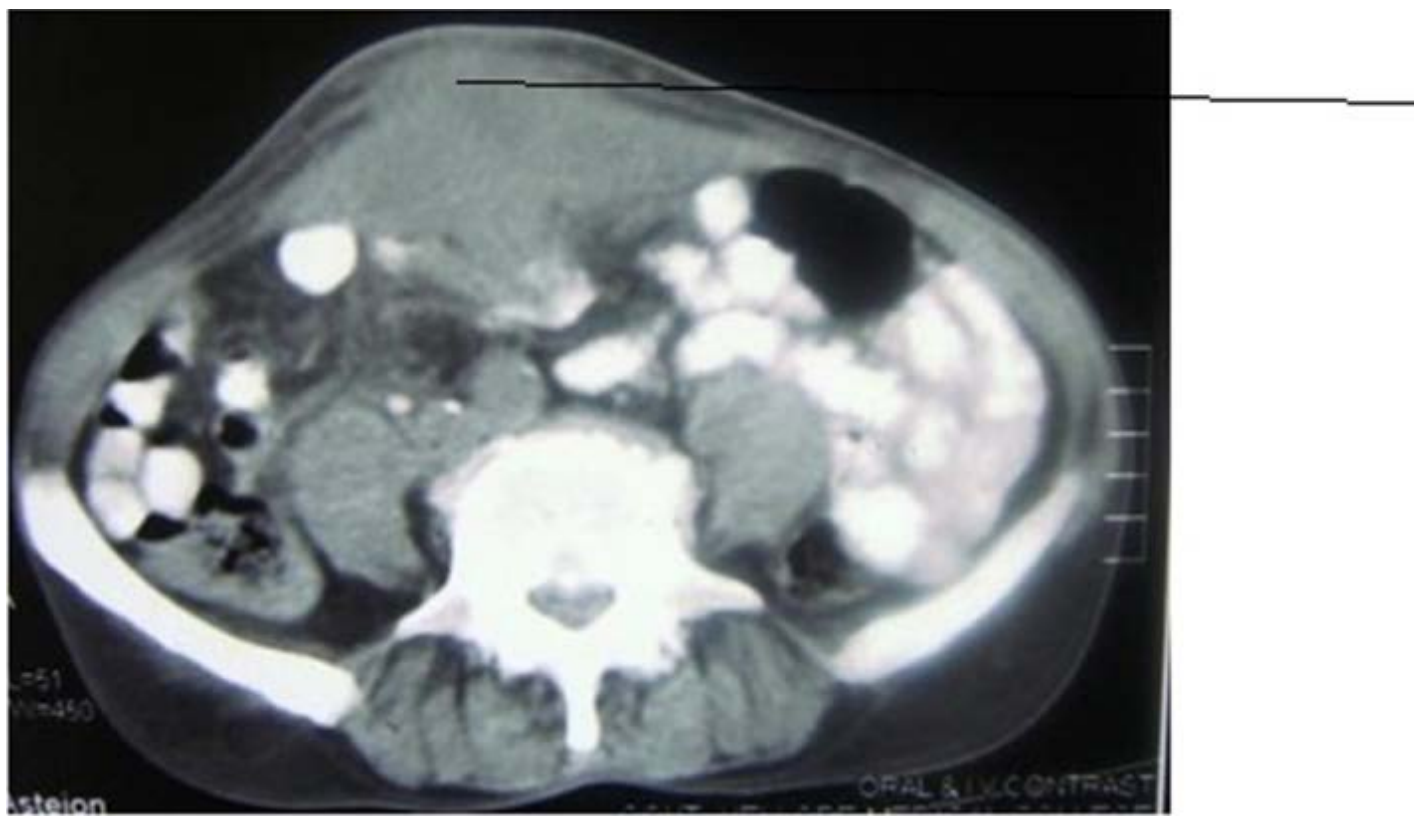

Figure 3. Histopathology showing - Panel A: mucin secreting adenocarcinoma and B: IHC showing negative PSA 


\section{Discussion}

Urinary bladder cancer is the second most frequent tumor of the genitourinary tract. [1] Adenocarcinoma of the urinary bladder is classified according to its origin into 3 categories: primary, urachal and metastatic. [2,3] The incidence of primary adenocarcinoma is $0.5-2 \%$. [4] However, incidence goes upto 5-11.4\% where bilharziasis is endemic. [5] The primary mucinous adenocarcinoma of the bladder is an extremely rare urologic entity, which is found in less than $2 \%$ of all urinary bladder tumors and is often presented as metastatic. it is often difficult to morphologically distinguish them from metastatic colorectal adenocarcinoma. The majority of primary adenocarcinomas of the urinary bladder (50-60\%) arise at the bladder base and remaining are associated with urachal remnants. [6] In case of nonurachal tumor male female ratio is $3: 1$ in contrast to urachal tumor which is $1: 1$. The incidence of this cancer increased with age with peaks in sixth, seventh and eight decades. Many authors suggest that adenocarcinomas arise through a process of intestinal metaplasia stimulated by chronic irritation. Among the other factors associated with urothelial adenocarcinoma, ectopia vesicae and persistent urachal remnants are the most common. Although adenocarcinomas arising in areas of urachal remnants differ clinically from those occurring at the bladder base, these neoplasms are similar in their pathology and behaviour. Adenocarcinomas of the urinary bladder, regardless of site, include the following histologic variations: 1) Adenocarcinoma non otherwise specified, 2) Adenocarcinoma of enteric type, 3) Adenocarcinoma with signet ring cells, 4) Mucinous adenocarcinoma, 5) Clear cell adenocarcinoma, 6) Hepatoid adenocarcinoma, 7) Mixed adenocarcinoma. [7] The usual malignant tumor is a well-to-moderately differentiated adenocarcinoma, secreting variable amounts of mucin. The tumor cells represent a combination columnar and goblet cells [8].

Hematuria is the most common presenting symptom,which is seen about $90 \%$ of patients. Almost half of the patients complain about dysuria, nocturia, frequency and pain. Cystoscopically, bladder adenocarcinomas ordinarily appear as single, nodular tumours that cannot be reliably distinguished from urothelial neoplasms.

Mucinous adenocarcinoma of the urinary bladder include large lakes of extracellular mucin mixed with collections of tumour cells. By definition, these mucinous foci should constitute at least half of the tumour mass. In some cases, there is an admixture of extracellular and intracellular mucin; the latter resulting in signet ring configuration [9].

Regarding immunohistochemistry, adenocarcinoma of the urinary bladder expresses CEA, CDX-2, MUC-1, MUC-2 and MUC-3, same as colonic adenocarcinoma. Cytokeratins 7 and 20 are positive, in contrast with colonic adenocarcinoma that expresses cytokeratin 20 but not cytokeratin 7 [10].

The differential diagnosis includes metastatic colonic adenocarcinoma, metastatic prostate cancer, urothelial neoplasms with glandular differentiation, intestinal metaplasia and nephrogenic metaplasia. Metastatic adenocarcinoma is differentiated using the immunophenotype (CK7 negative and CK 20 positive).PSA is positive in case of metastatic prostate carcinoma. Urothelial neoplasm with glandular differentiation may contain intracellular and luminal mucins; however, mucins are not abundant. In addition, in this type of carcinoma, signet-ring cells are not prominent and the "glands" are surrounded by pseudostratified epithelium. Intestinal metaplasia may infiltrate the lamina propria or even the bladder wall. Mucinous lakes are not uncommon in these cases and their presence in a tissue sample is diagnostic of adenocarcinoma only with the presence of neoplastic cells. The cells of intestinal metaplasia lack nuclear anaplasia and rarely involve the muscularis propria. Nodular areas of cystitis glandularis rich in goblet cells should be considered benign, even if the nodules extend into the lamina propria.

Prognosis varies with stage, as mucinous adenocarcinoma mostly presents in advanced state, prognosis is poor in this cancer. Patients with urachal tumours tend to have a better short-term survival rate than those with nonurachal cancers [11].

\section{References}

[1] Grossfeld GD, Carroll PR (1998): Evaluation of asymptomatic microscopic hematuria. Urol Clin North Am 25:661-676.

[2] Dahm P, Gschwend JE (2003): Malignant non-urothelial neoplasms of the urinary bladder: a review. Eur Urol 44:672-681.

[3] Wheeler J.D. and Hill W.T (1954): Adenocarcinoma involving the urinary bladder. Cancer, 7: 468-76.

[4] Thomas D.C., Ward A.M. and Williams J.L (1971): A study of 52 cases of adenocarcinoma of the bladder. Br. J.Urol., 43: 4-15.

[5] El-Bolkainy M.N., Mokhtar N.M., Ghoneim M.A. and Hussein M.H (1981): The impact of schistomiasis on the pathology of bladder carcinoma. Cancer, 48: 2643-8.

[6] Mazzucchelli R, Scarpelli M, Montironi R (2003): Mucinous adenocarcinoma with superficial stromal invasion and adenoma of urachal remnants: a case report. J Clin Pathol, 56:465-467.

[7] Eble JN, Epstein JI, Seternhenn IA (2004): World Health Organization Classification of Tumours. Pathology and Genetics, Tumours of the Urinary System and Male Genital track. Lyon: IARC Press, 128-132.

[8] Murphy WM, Grignon DJ, Periman EJ (2004): Tumors of the Kidney, Bladder and Related Urinary Structures. American Registry of Pathology, New York, 4; 304-309.

[9] Marques ML, D’Alessandro GS, Chade DC, Lanzoni VP, Saiovici S, Ramos de Almeida JR (2007): Primary mucinous adenocarcinoma of the bladder with signet-ring cells: case report. Sao Paulo Med J, 125:297-299.

[10] Bostwick DG, Cheng L (2008): Urologic Surgical Pathology. Adenocarcinoma of the Urinary Bladder. Elsevier, New York, 300302.

[11] Stenhouse G, Mcrae D, Pollock AM (2003): Urachal adenocarcinoma in situ with pseudomyxoma peritonei: a case report. J Clin Pathol, 56:152-153. 